Review Article

\title{
Association of Diet, Quality of Life and Premenstrual Syndrome; A Review
}

\author{
Quratulain Javaid \\ - - - - - - - - - - - - - - - - - - - - - - - - - - - - - - - -
}

\begin{abstract}
Premenstrual syndrome is a disorder experienced by the females of reproductive ages. The etiology of this common condition is unknown. The presentation of symptoms is highly variable all over the world. The variability in the presentation of symptoms could be linked to social and cultural effects of environment. There can be psychological, physical or behavioral characteristic manifestations linked to premenstrual syndrome. The presentation of premenstrual syndrome can effect a female's life. Household chores, work place tasks and interpersonal relationships are effected gravely. Dietary intake of the individuals is associated with the symptoms of premenstrual syndrome symptoms. Intake of diet loaded with whole grains, fish, fruits, vegetables, micronutrients like vitamin B1, D and E have shown to have protective effects against the development and severity of premenstrual syndrome symptoms. Intake of harmful food items like refined sugar, fast food, sweetened drinks, alcohol and chocolates are associated with increase in severity of the condition.
\end{abstract}

Key words: Premenstrual syndrome, prevalence, diet, quality of life

\section{$\overline{\text { How to }}$ cite this Article:}

Javaid Q. Association of Diet, Quality of Life and Premenstrual Syndrome; A Review. J Bahria Uni Med Dental Coll. 2020; 10(3): 234-8 DOI: https://doi.org/ 10.51985/JBUMDC2020028

\section{INTRODUCTION:}

Menstrual cycle has characteristics that are variable among different females. Premenstrual syndrome (PMS) is a condition that is associated with certain physical and psychological and behavioral conditions. The term of premenstrual syndrome was first coined by Frank in the year 1931 after he observed females having certain symptoms that were relieved after the start of menstruation. It occurs in the end of luteal phase of the menstrual cycle. ${ }^{1}$ It gets relieved after 3 days of the menstrual cycle. ${ }^{2}$ The etiology of post menstrual syndrome is not known as yet. ${ }^{1}$

The symptoms include edema, increased weight and nervousness. ${ }^{3}$ There are wide range of symptoms that can include both the psychiatric as well as somatic symptoms. ${ }^{4}$ Premenstrual syndrome is associated with symptoms like bloating, muscular pain, anxiety, tetchiness, breast edema, tenderness in the breast, increase sleep or absence of sleep, increase appetite/craving of food, weight gain and mood fluctuations. ${ }^{5}$ According to published standard guidelines, any one symptom form the affective category and one from the somatic category should be present in each of the three menstrual cycles that have been occurred previously to declare a female having premenstrual syndrome. ${ }^{6}$

Various researches have documented different reasons for the appearance of premenstrual syndrome. Diet, genetic factors, way of living have been identified as important factors which contribute in inducing the symptoms of this

Quratulain Javaid
Senior Lecturer, Anatomy Department
Bahria University Medical and Dental College, Karachi
Email: docannie2010@gmail.com
Received: 06-Apr-2020
Accepted: 02-Jul-2020

condition. $^{7}$ There are various treatment options like antidepressants and serotonin reuptake inhibitors for premenstrual syndrome but the use of medicines and chemical agents has its own adverse effects. A study conducted in Qazvin University of Medical Sciences has documented that life style changes can help decrease the severity and occurrence of PMS. ${ }^{8}$ Dietary modification is considered to be one of the best available options in alleviating the symptoms of PMS. ${ }^{9}$

Modification in diet is documented to have an impact on the occurrence of symptoms of premenstrual syndrome. Reduction of high saturated food, refined carbohydrate and salt are observed to be beneficial in reducing the symptoms of this condition. Inclusion of certain macro and micronutrients like high fiber diet, unsaturated fats, vitamin $\mathrm{E}$ and $\mathrm{B}$ have revealed to be of high value in controlling the severity of the syndrome. ${ }^{10,11}$ Another study conducted on the females living in Sharjah has demonstrated that symptoms of the premenstrual syndrome aggravate when diet includes salt, high number of calories and increase content of fat. ${ }^{12}$

\section{Prevalence of premenstrual syndrome:}

Variable prevalence percentages are mentioned in the leterature. This could be due to the cultural and ethnic or genetic differences among the various nations of the world. ${ }^{13}$ A study published in 'Journal of International Dental and Medical Research' has stated that prevalence of post menstrual syndrome is $75 \%$ to $80 \%$ in the females which are in reproductive age. ${ }^{14}$ Corresponding findings in terms of prevalence of PMS was noted in a study conducted in Islamabad, Pakistan. Eighty percent of the respondents reported symptoms of premenstrual syndrome. An even higher percentage was mentioned in a study conducted in Sharjah. According to the authors, there was at least one symptom of premenstrual syndrome and it was there in $95 \%$ 
of the females. ${ }^{12}$ A study conducted in Karachi, Pakistan has documented that prevalence of PMS was $79.5 \%$ according to a self-reported questionnaire and $23.9 \%$ according to the guidelines given by American College of Obstetrics and Gynecology. ${ }^{5}$ A research conducted in Puducherry, India has documented the prevalence of premenstrual syndrome to be $62.7 \%$ in females of reproductive age groups. ${ }^{13}$ Similarly, a study conducted in Turkey has documented the prevalence of PMS to be $58.1 \% .{ }^{14}$ A study published in 'Brazilian Journal of Nutrition' has documented that among the study participants, PMS was present in $43.80 \%$ of individuals. ${ }^{15}$ On the contrary, Akbari in her research study has stated comparatively low percentage of premenstrual syndrome. According to her, $31 \%$ of the research participants studying at a medical college in Chitradurga, India experienced PMS. ${ }^{16}$ A study on secondary school students in Jeddah, Saudi Arabia has reported that one third of the research participants experienced premenstrual syndrome. ${ }^{17}$

\section{Variable symptoms of premenstrual syndrome}

Numerous researches have documented variability in the appearance of symptoms of premenstrual syndrome. Some studies reported physical symptoms to be more common than other symptoms. A study conducted in India, has reported muscle, joint and backache to be among the most common symptoms experienced by the participants followed by abdominal pain and bloating. ${ }^{13}$ Similarly, a study conducted in Turkey has documented physical symptoms to be among the most common ones other being the psychological symptoms. Among the physical symptoms, the most common was backache that was present in $73.6 \%$ of the individuals. Backache was followed by pain in the pelvis $(61.3 \%)$, tiredness (55.7\%), irritable mood (52.5\%), bloating (497\%), change in appetite (43.4\%), tenderness in the breast (39.6\%) and episodes of depression (34.9\%). ${ }^{15}$ As reported by a study on medical students, physical symptoms were more as compared to the psychological and behavioral symptoms. The symptoms were reported to be $95 \%, 75 \%$ and $43 \%$ respectively. Among the physical symptoms, the most common one was breast tenderness (60\% of participants) followed by headache (40\%), muscle and joint pain (30\%), bloating (20\%) and increase in weight (20\%). Among the psychological symptoms, the most common was depression (36\%), followed by anxiety (30\%), sleep pattern changes (25\%), guilt feeling (20\%) and tearfulness (20\%). Changes in behavior pattern was divided into two categories. Low academic performance was present in $76 \%$ and social withdrawal was noted in $46.7 \%$ of the participants. ${ }^{18}$

Many researches have reported psychological symptoms to be more common than others have. A study conducted on the medical students of Karachi, Pakistan has documented tetchiness to be the commonly occurring symptom (81.7\%). Other reported characteristics were anger (66.9\%), depressive mood changes (53.1\%), anxiety (46.9\%), skin issues (42.7\%), tenderness of breast (39.6\%), gastrointestinal issue (39.3\%), social withdrawal (37.1\%), swelling of the abdomen $(33.7 \%)$, sleep pattern changes $(33.4 \%)$, headache $(21.9 \%)$, and arm/leg swellings $(8.1 \%) .{ }^{5}$ Variability in symptoms presentation was also noted in a research study conducted in Sharjah. The most common symptom mentioned was depression $(95 \%)$. Other reported symptoms were lack of energy (92\%), body pain (89.3\%), angry mood $(85.7 \%)$ and food carvings (84.7\%). Behavioral symptoms were the least common as compared to the psychological and the physical symptoms. ${ }^{12}$

\section{Disruption of quality of life}

The premenstrual syndrome can have variable effects on the females' lives. Health related quality of life among young females of reproductive ages is effected gravely by the symptoms of PMS. ${ }^{19}$ According to a research study, young girls' work efficiency, concentration and quality of work are affected by the condition adversely. Premenstrual syndrome has a disturbing effect on the females. ${ }^{14}$ Similarly, a recent study has reported that PMS can adversely affect females' public as well as routine life. The work life atmosphere as well as social life of the female gets disturbed. ${ }^{17}$ A study conducted in Karachi, Pakistan has reported that among participants, $60.4 \%$ have reported that their normal routine life gets disrupted due to PMS. ${ }^{5}$ A study conducted on Turkish nurses has mentioned that stress was observed to be found more in those having PMS. ${ }^{20}$ It was documented in an Egyptian research that because of the premenstrual syndrome, occupational work was negatively affected. ${ }^{21}$

Other than the work life, sexual life of a female is also disturbed. A study conducted in Iran has reported that there are various unfavorable effects of PMS on a woman's life. It can effect physical, behavioral and psychological health. The sexual life and henceforth relationship between couples also gets disrupted because of the severity of symptoms. Agitation is one of the features that can lead to social distancing among the females of reproductive ages. ${ }^{22}$

Premenstrual syndrome seriously effects the concentration and hence learning capabilities of the students. A study conducted on Thai high school students has documented that premenstrual syndrome effects academics. The students experiencing the symptoms of PMS have difficulty in concentrating in the classrooms. Other than that, interpersonal skills are also affected and collaborative work cannot be done in an effective manner. All these factors contribute to low academic scores. ${ }^{23}$

\section{Knowledge about the prevention of PMS}

In the present day society, females over the world have different strategies to cope up with the symptoms of premenstrual syndrome. A study conducted in Islamabad, Pakistan has reported that $71.5 \%$ of the study participants had no clue about the prevention options available for PMS. Out of the $29.5 \%$ who had idea about the prevention of PMS, 50\% believed that home remedies could prevent the 
symptoms. 30\% believed that supportive measures like reassurance play a part while $20 \%$ thought that medications could be helpful. ${ }^{18}$ A research study conducted in Karachi, Pakistan has notified that several treatment options were adopted by the study participants. The most commonly used treatment was painkillers (41\%), followed by homeopathic medicine (1.4\%), supplements containing vitamins $(5.1 \%)$, traditional remedies $(13.8 \%)$, exercise $(17.7 \%)$, antidepressants $(0.3 \%)$ while $49.4 \%$ did nothing for the symptoms. The study revealed that $77.5 \%$ of the participants considered it an important issue and they were of the opinion that it should be discussed. ${ }^{5}$

\section{Diet helpful in prevention and severity of PMS}

There are various research studies that have documented the effect of food intake and its role in development as well as severity of premenstrual syndrome. In a recent study, different vegetables were compared for their effects on PMS. It was mentioned that cruciferous vegetables (cabbages, broccoli and cauliflower) intake is associated with protective effect against the development of PMS. Vegetables like green (spinach, basil, parsley etc.), dark yellow (yellow squash, carrot etc.) and other categories do not have a significant effect on the symptoms of premenstrual syndrome.${ }^{24} \mathrm{~A}$ study has mentioned the protective effects of fruits in alleviating the behavioral, psychological and physical symptoms of PMS. The reason could be the antioxidants role which are there in enormous amounts in the fruits. ${ }^{12} \mathrm{~A}$ result of a triple blind control trial conducted in Iran has concluded that intake of vitamin B1 in diet can prevent physical symptoms of PMS. According to the study, calcium intake can help prevent both psychological and physical symptoms. ${ }^{25}$ A study conducted in Japan has documented that PMS can be relieved by inclusion of fish in the diet. ${ }^{26}$ A study published in 'Public Health Nutrition', has documented that diet rich in eggs, tomatoes, meat, fruits, condiments and nuts is associated with alleviating the symptoms of PMS. ${ }^{27}$ Bahrami has reported that intake of vitamin $\mathrm{D}$ is associated with protective effects that can help prevent symptoms of premenstrual syndrome. ${ }^{28}$ Results of a double blind controlled trial in Iran, has stated that Vitamin $\mathrm{D}$ and $\mathrm{E}$ are both beneficial in relieving the symptoms of premenstrual syndrome. ${ }^{29}$ A randomized controlled trial published in 'British Journal of Nutrition', has documented that diet has an effect on the symptoms of premenstrual syndrome. Diet rich in grains can have beneficial effects on the symptoms of this condition. The inclusion of whole grains in the diet were found to have a beneficial effect in preventing and reducing the severity of PMS. Significant decrease in mood related symptoms $(\mathrm{p}<0.01)$ and highly significant improvement in behavioral $(\mathrm{p}<0 \cdot 003)$, physical $(\mathrm{p}<0 \cdot 001)$ and general symptoms $(\mathrm{p}<0 \cdot 001)$ was mentioned $^{30}$ On the contrary, in a research conducted on students of Sharjah, it was documented that complex carbohydrates do not have any effects on Premenstrual syndrome. ${ }^{12}$

\section{Diet causing premenstrual syndrome}

Researches across the world have shown variable food items that contribute in the occurrence of premenstrual syndrome. A study in Turkey has revealed that alcohol, fast food, two or more cups of caffeine are associated with the development of premenstrual syndrome. It was also documented that breakfast skippers are also associated with PMS symptoms. ${ }^{31}$ A research study has stated that while a female is having PMS, she develops the craving to eat sweetened foods. ${ }^{32} \mathrm{~A}$ study conducted on the college girls has documented that food items like caffeine, junk food and high sugar content food contribute in producing the symptoms. ${ }^{13}$ Parallel findings were reported in a study conducted in Sharjah. In the luteal phase of the cycle the females had craving for sweets like Baklava, Kunafa, chocolates and other sweetened food items. ${ }^{12}$ Comparable findings were mentioned in a study conducted on the university students. The students with PMS have the habit of consuming refined sugar, fatty meals, fast food and chocolates. The students' diet was lacking essential vitamins and minerals. ${ }^{15} \mathrm{~A}$ case control study has reported analogous findings. The western diet which includes burgers, soft drink and processed meat contributes in the development of PMS with highly significant $p$ value of $<0.001 .^{27}$

Various studies have observed the effects of caffeine in the diet. A research has documented that caffeine in the diet not only cause the PMS but also prolongs its duration. It was recommended that a week before the start of menstruation the consumption of the caffeine should be decreased or finished. ${ }^{33}$ Another study conducted in Manipal, has reported that intake of caffeine contributes in the aggravation of symptoms of PMS. ${ }^{34}$ Contradictory results were documented in a prospective study published in 'American Journal of Clinical Nutrition'. The research reported that caffeine intake does not effect premenstrual syndrome..$^{35}$ Likewise, another research has documented that caffeine was not shown to have an effect on the PMS symptoms. ${ }^{12}$ A quasi-experimental study conducted in India, has reported beneficial effects of calcium intake in decreasing the effects of PMS. ${ }^{36}$

\section{CONCLUSION:}

Premenstrual syndrome is a common condition that is experienced by majority of females in reproductive age group all around the world. The prevalence of PMS varies among females belonging to different regions of the world. The presentation of symptoms is highly variable. There can be psychological, physical or behavioral features linked to premenstrual syndrome. Dietary intake of the individuals have been linked to the premenstrual syndrome symptoms. Consumption of diet loaded with whole grains, fish, fruits, vegetables, micronutrients like vitamin $\mathrm{B} 1, \mathrm{D}$ and $\mathrm{E}$ have protective effects against the development and severity of premenstrual syndrome symptoms. Intake of harmful food items like refined sugar, increased salt, fast food, sweetened 
drinks, alcohol and chocolates are associated with increase in severity of the condition.

Author Contribution:
Quratulain Javaid: 1 . Substantial contributions to conception
and design, or acquisition of data, or analysis and interpretation
of data
2. Drafting the article or revising it critically for important
intellectual content
3. Final approval of the version to be published
4. Agreement to be accountable for all aspects of work in
ensuring that questions related to accuracy or integrity of any
part of the work are appropriately investigated and resolved.

\section{REFERENCES:}

1. Jehan S, Auguste E, Hussain M, Pandi-Perumal SR, Brzezinski A, Gupta R, Attarian H, Jean-Louis G, McFarlane SI. Sleep and premenstrual syndrome. Journal of sleep medicine and disorders. 2016;3(5):1061-79

2. Acikgoz A, Dayi A, Binbay T. Prevalence of premenstrual syndrome and its relationship to depressive symptoms in firstyear university students. SMl. 2017;38(11):1125-31.

3. Ellen RM. A Survey of Multidimensional and Interdisciplinary Approaches to Premenstrual Syndrome. InMenstruation, Health And Illness 2019 May 30 (pp. 129-142). Taylor \& Francis. Abdollahi R, Abiri B, Sarbakhsh P, Kashanian M, Vafa M. The Effect of Vitamin D Supplement Consumption on Premenstrual Syndrome in Vitamin D-Deficient Young Girls: A Randomized, Double-Blind, Placebo-Controlled Clinical Trial. Complement Med Res. 2019;26(5):335-41.

4. Mohib A, Zafar A, Najam A, Tanveer H, Rehman R. Premenstrual syndrome: existence, knowledge, and attitude among female university students in Karachi. Cureus. 2018;10(3):1-10.

5. Hofmeister S, Bodden S: Premenstrual syndrome and premenstrual dysphoric disorder. Am Fam Physician. 2016;94:236-40.

6. Matsumoto T, Asakura H, Hayashi T. Biopsychosocial aspects of Premenstrual Syndrome and premenstrual dysphoric disorder. Gynecol Endocrinol. 2013;29(1):67-73 http://dx.doi.org/10.3109/09513590.2012.705383

7. Mohebbi M, Akbari SA, Mahmodi Z, Nasiri M. Comparison between the lifestyles of university students with and without premenstrual syndromes. Electronic physician. 2017;9(6):448996

8. Malik R, Bhat MD. The management of Premenstrual syndrome: A review. BJMS. 2018;17(1):16-20.

9. Cheng SH, Shih CC, Yang YK, Chen KT, Chang YH, Yang YC. Factors associated with Premenstrual Syndrome: A survey of new female university students. Kaohsiung J Med Sci. 2013;29(2):100-5.

10. Vaziri F, Hoseini A, Kamali F, Abdali K, Hadianfard M, Mehrab S. Comparing the effects of aerobic and stretching exercises on the ýntensity of primary dysmenorrhea in the students of universities of Bushehr. J Family Reprod Health. 2015;9(1):23-8.

11. Hashim MS, Obaideen AA, Jahrami HA, Radwan H, Hamad HJ, et.al. Premenstrual Syndrome Is Associated with Dietary and Lifestyle Behaviors among University Students: A CrossSectional Study from Sharjah, UAE. Nutrients. 2019;11(8): 1939-57.
12. Yilmaz-Akyuz E, Aydin-Kartal Y. The effect of diet and aerobic exercise on Premenstrual Syndrome: Randomized controlled trial. Revista de Nutrição [Internet]. 2019 [cited 30 March 2020];32:1-10. Available from: https://doi.org/10. 1590/1678-9865201932e180246.

13. Juber MB, Sunariani J. Sweet Taste Sensitivity and Its association with Serum Zinc Levels in Women with Premenstrual Syndrome. Journal of International Dental and Medical Research. 2017;10(2):354.

14. Bhuvaneswari, K., Rabindran, P. and Bharadwaj, B. (2019). Prevalence of premenstrual syndrome and its impact on quality of life among selected college students in Puducherry. Natl. Med. J. India., 32(1): 17-19.

15. Acikgoz. A., Dayi, A. and Binbay, T.(2017). Prevalence of premenstrual syndrome and its relationship to depressive symptoms in first-year university students. SMJ., 38(11): 1125-31.

16. Akbari R, Sudharani, Kallupurackal SJ, Gowda N, Suryakantha AH. Prevalence of Premenstrual Syndrome among Medical Students. NJCM. 2017;8(6):292-4.

17. Salem IM, Alsamti MY, Murad MA. Predictors of Premenstrual Syndrome among Female Students at Governmental Secondary Schools in Jeddah, Saudi Arabia: A Cross-sectional Study. EJHM. 2020;78(2):337-47.

18. Kalsoom U, Sultana A, Amjad T, Bairam S. Prevalance of premenstrual syndrome and knowledge assessment regarding it's prevention among medical students of a private medical college of Islamabad. PAFMJ. 2018;68(1):159-64.

19. El Fotooh A, Fawzi H, El Din N, Ali S, Gonied AS. A Premenstrual Syndrome and Its Association with Adolescent Girls Quality Of Life. ZUMJ. 2018;14(1):190-202.

20. Sut HK, Mestogullari E. Effect of premenstrual syndrome on work-related quality of life in Turkish nurses. Saf health Work. 2016;7(1):78-82.

21. Montazeri A, Taghizadeh Z, Taheri S, Siahbazi S, Masoomi R. Domination of premenstrual syndrome on women's quality of life: a qualitative study. Payesh (Health Monitor). 2019;18(1):53-66

22. Mohamed SM. Premenstrual syndrome and quality of life among Sohag University female students. Assiut Scientific Nursing Journal. 2018;6(13):108-15.

23. Buddhabunyakan N, Kaewrudee S, Chongsomchai C, Soontrapa S, Somboonporn W, Sothornwit J. Premenstrual syndrome (PMS) among high school students. Int. J. Women's Health. 2017;9:501-5.

24. Babakhani K, Sotoudeh G, Siassi F, Qorbani M. Comparison of Vegetable Intake in Nurses with and without Premenstrual Syndrome: A Case-Control Study. SEMJ. 2019;21(2).e91319

25. Samieipour S, Kiani F, BabaeiHeydarabadi A, Tavassoli E. Comparing the effects of vitamin B1 and calcium on premenstrual syndrome (PMS) among female students, IlamIran. Int. J. Pediatr. 2016;4(9):3519-28.

26. Takeda T, Imoto Y, Nagasawa H, Takeshita A, Shiina M. Fish consumption and premenstrual syndrome and dysphoric disorder in Japanese collegiate athletes. J PEDIATR ADOL GYNEC. 2016;29(4):386-9.

27. Moradifili B, Ghiasvand R, Pourmasoumi M, Feizi A, Shahdadian F, Shahshahan Z. Dietary patterns are associated with premenstrual syndrome: evidence from a case-control study. Public Health Nutr. 2019;15:1-0. 
28. Bahrami A, Avan A, Sadeghnia HR, Esmaeili H, Tayefi M, Ghasemi F, et al. High dose vitamin D supplementation can improve menstrual problems, dysmenorrhea, and premenstrual syndrome in adolescents. GYNECOL ENDOCRINOL. 2018;34(8):659-63.

29. Dadkhah H, Ebrahimi E, Fathizadeh N. Evaluating the effects of vitamin D and vitamin E supplement on premenstrual syndrome: A randomized, double-blind, controlled trial. IJNMR. 2016;21(2):159-64.

30. Esmaeilpour M, Ghasemian S, Alizadeh M. Diets enriched with whole grains reduce premenstrual syndrome scores in nurses: an open-label parallel randomised controlled trial. Br. J. Nutr. 2019;121(9):992-1001.

31. Isik H, Ergöl S, Aynioglu Ö, Sahbaz A, Kuzu A, Uzun M. Premenstrual syndrome and life quality in Turkish health science students. TURK J MED SCI. 2016;19;46(3):695-701.
32. Sherehan RA, Galal AE, Soad A R, Eman MA. Effect of Caffeinated Beverages Intake on Premenstrual Syndrome among Nursing Students. EJHC. 2018;9(2):61-74.

33. Sharma P, Patro A, Ibrahim S, Reddy T, Jain N, Mallya SD. Premenstrual symptoms and lifestyle Factors Associated with it among Medical students. Indian J Public Health Res Dev. 2018;9(10):39-45.

34. Purdue-Smithe AC, Manson JE, Hankinson SE, BertoneJohnson ER. A prospective study of caffeine and coffee intake and premenstrual syndrome. Am. J. Clin. Nutr. 2016;104(2):499-507

35. Bharati M. Comparing the effects of yoga \& oral calcium administration in alleviating symptoms of premenstrual syndrome in medical undergraduates. Int. J. Caring Sci. 2016;5(3):179-85 